\title{
Phase synchronization in an array of driven Josephson junctions
}

\author{
Chitra $\mathrm{R}$ N* and V C Kuriakose $\oplus^{\dagger}$ \\ Department of Physics, Cochin University of Science and Technology, Kochi, 682022
}

(Dated: September 1, 2021)

\begin{abstract}
We consider an array of $\mathrm{N}$ Josephson junctions connected in parallel and explore the condition for chaotic synchronization. It is found that the outer junctions can be synchronized while they remain uncorrelated to the inner ones when an external biasing is applied. The stability of the solution is found out for the outer junctions in the synchronization manifold. Symmetry considerations lead to a situation wherein the inner junctions can synchronize for certain values of parameter. In the presence of a phase difference between the applied fields, all the junctions exhibit phase synchronization. It is also found that chaotic motion changes to periodic in the presence of phase differences.
\end{abstract}

PACS numbers: 05.45.Xt, 05.45.Gg

*Electronic address: rchitra@cusat.ac.in

${ }^{\dagger}$ Electronic address: vck@cusat.ac.in 
Due to the application of chaotic synchronization in secure communication to brain modeling a great deal of investigation has been done in this field. The presence of even a small phase difference between the applied fields was found to desynchronize a completely synchronized system. Also the phase difference was found to have application in taming chaos in dynamical systems. Recently it was observed that the end lasers in an array of three laser system was found to synchronize while it remained uncorrelated with the middle laser which originally connected the two. In this work we study an array of Josephson junctions in the presence a phase difference between the driving fields and its effect on synchronization and suppression of chaos.

\section{INTRODUCTION}

Chaos in Josephson junction (JJ) has been studied extensively after its presence was demonstrated using numerical simulation [1]. When we treat JJs within the StewartMcCumber model, the equation describing the behaviour of JJ is identical to the equation for a driven damped pendulum which has been studied theoretically for several routes to chaos [2, 3]. Thus JJ becomes an ideal physical system to study chaos. The rf- biased JJs find practical importance in the construction of devices like parametric amplifiers, voltage standards, pulse generators, SQUID for detection of very weak magnetic fields, etc. [4, 5, 6]. For these devices, it is essential to avoid all types of noise, chaos etc. JJs consisting of Superconductor-Insulator-Normal metal-Insulator-Superconductor (SINIS) showing nonhysteretic I-V characteristics with high damping has been fabricated for programmable dcvoltage standards [7] or ac-voltage standards based on synthesis of calculable wave forms [8].

Pecora and Carroll in 1990 reported that synchronization of chaotic systems [9] could be achieved, since then different types of synchronization such as complete, generalized and phase synchronization of chaotic oscillators have been described theoretically and ob-

served experimentally [10, 11]. Synchronized chaotic oscillations have been found in many nonlinear systems like lasers, neural network, etc[12, 13]. Chaotic synchronization also find application in communication. It was demonstrated using Rössler oscillators that during the transmission of information about a stimulus through an active array, the stimulus created the way to be transmitted by making the chaotic elements to phase synchronize [14]. The 
stability of synchronous state is analyzed by Lyapunov function method [15] and the master stability approach [16]. Phase difference between the applied fields plays an important role in suppressing chaos and the synchronization of chaotic systems. Duffing oscillator was studied for the effect of phase difference on chaotic synchronization [17]. Josephson junction has been investigated for both periodic and chaotic synchronization. Coupling between self generated Josephson oscillations through a microwave transmission line was found to play an important role in collective synchronization of JJ array [18]. In a system of two JJs in parallel, the phase difference between the applied fields was found to bring chaotic motion to a periodic one for a large range of parameter values [19]. A parallel array of coupled short JJs linked together by inductors has been used to fabricate highly sensitive detectors [20]. Although there is extensive work on synchronization of coupled JJs, studies on chaotic synchronization of JJs is much less.

In this work we analyze a parallel array of N-coupled JJs with parameters lying in the chaotic regime and study synchronization of the system. The paper is organized as follows. In section II we discuss the model for an array of JJs linked in parallel with linking resistor $R_{s}$ in between. Section III contains the study of the synchronization in such an array and discuss the stability of the synchronous solution. The effect of phase difference between the applied fields on synchronization and its role in suppressing chaos is also discussed. Results are summarized in section IV.

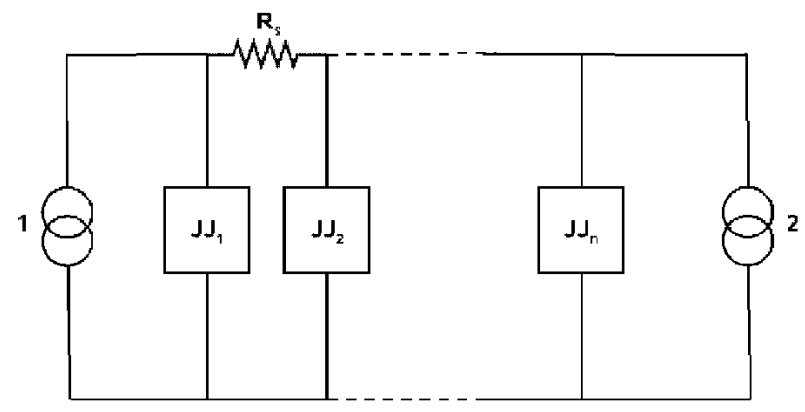

FIG. 1: Schematic representation of an array of JJ linked in parallel with a linking resistor $R_{s} .1$ and 2 are the driving fields. 


\section{THE MODEL}

The equation of a single Josephson junction represented by the resistively and capacitively shunted junction (RCSJ) model can be written by solving Kirchoff's law as

$$
\frac{\hbar C}{2 e} \frac{d^{2} \phi}{d t^{\prime 2}}+\frac{\hbar}{2 e R} \frac{d \phi}{d t^{\prime}}+i_{c} \sin \phi=i_{d c}^{\prime}+i_{0}^{\prime} \cos \left(\omega t^{\prime}\right)
$$

where $\phi$ is the phase difference of the wave function across the junction, $i_{0}^{\prime} \cos \left(\omega t^{\prime}\right)$ is the driving rf - field and $i_{d c}^{\prime}$ is the dc bias. The junction is characterized by a critical current $i_{c}$, capacitance $C$ and normal resistance $R$. The coupled JJ considered here consists of a pair of such junctions wired in parallel with a linking resistor $R_{s}$ [21]. A schematic representation of an array of JJ wired in parallel with linking resistors is given in fig 1. The equation of motion for an array of $\mathrm{N}$ coupled current driven JJs can be written in the normalized form as

$$
\begin{array}{ccc}
\ddot{\phi}_{1}+\beta \dot{\phi}_{1}+\sin \phi_{1} & =i_{d c}+i_{0} \cos (\Omega t)-\alpha_{s}\left[\dot{\phi}_{1}-\dot{\phi}_{2}\right] \\
\vdots & \vdots & \vdots \\
\ddot{\phi}_{i}+\beta \dot{\phi}_{i}+\sin \phi_{i} & =\alpha_{s}\left[\dot{\phi}_{i+1}+\dot{\phi}_{i-1}-2 \dot{\phi}_{i}\right] \\
\vdots & \vdots & \vdots \\
\ddot{\phi_{N}}+\beta \dot{\phi_{N}}+\sin \phi_{N} & = & i_{d c}+i_{0} \cos (\Omega t)-\alpha_{s}\left[\dot{\phi}_{N}-\dot{\phi}_{N-1}\right]
\end{array}
$$

where $i$ varies from 2 to $\mathrm{N}-1$ and the dimensionless damping parameter $\beta$ is defined as

$$
\beta=\frac{1}{R} \sqrt{\frac{\hbar}{2 e i_{c}}} .
$$

The normalized time scale is written as $t=\omega_{J 1} t^{\prime}$ where $\omega_{J 1}=\left(2 e i_{c 1} / \hbar C_{1}\right)^{\frac{1}{2}}$. The dc bias current $i_{d c}^{\prime}$ and the rf amplitude $i_{0}^{\prime}$ are normalized to the critical current $i_{c 1}$. The actual frequency $\omega$ is re-scaled to $\Omega=\omega / \omega_{J 1}$ and the coupling factor is defined as $\alpha_{s}=\left(R_{1} / R_{s}\right) \beta$.

The Josephson junction is chaotic for the parameter values $\beta=0.3, i_{0}=1.2, \omega=0.6$ and $i_{d c}=0.3$. We fix these parameter values for the numerical simulations. The junctions are taken to be identical and for a coupling strength of $\alpha_{s}=0.37$, the outer junctions synchronize while the inner junction remain uncorrelated with the two outer ones. It can be seen from Fig 2(a) that the outer junctions are synchronized whereas Fig!2(b) shows that it is uncorrelated with the middle junction for an array of three JJs. 


\section{STABILITY ANALYSIS}

In order to perform the stability analysis for the synchronized state of N-coupled Josephson junctions, we first consider three JJs linked in parallel. In the first order form the three identical junctions can be written as

$$
\begin{aligned}
& \dot{\phi}_{1}=\psi_{1} \\
& \dot{\psi_{1}}=-\beta \psi_{1}-\sin \phi_{1}+i_{d c}+i_{0} \cos (\Omega t)-\alpha_{s}\left[\psi_{1}-\psi_{2}\right] \\
& \dot{\phi}_{2}=\psi_{2} \\
& \dot{\psi}_{2}=-\beta \psi_{2}-\sin \phi_{2}+\alpha_{s}\left[\psi_{1}+\psi_{3}-2 \psi_{2}\right] \\
& \dot{\phi}_{3}=\psi_{3} \\
& \dot{\psi}_{3}=-\beta \psi_{3}-\sin \phi_{3}+i_{d c}+i_{0} \cos (\Omega t+\theta)-\alpha_{s}\left[\psi_{3}-\psi_{2}\right]
\end{aligned}
$$

From eq $3 \mathrm{a}$ and $3 \mathrm{c}$ it can be observed that the outer junctions are identical and symmetric with interchange of variables in the absence of a phase difference $\theta$ between the applied fields. Hence there exists an identical solution for the outer systems given by $\phi_{1}=\phi_{3}=\phi(t)$ and this type of behavior where systems show identical behavior is called complete synchronization. Due to asymmetry the middle junction may have different dynamics. The stability of the synchronous solution of the outer junctions is analyzed by two methods.

We define the difference variables $\phi_{13}^{-}=\frac{\phi_{1}-\phi_{3}}{2}$ and $\psi_{13}^{-}=\frac{\psi_{1}-\psi_{3}}{2}$ and the approximate dynamics transverse to the synchronization manifold is obtained by linearizing the corresponding subsystem consisting of the outer junctions. The equation may be given as

$$
\begin{aligned}
& \dot{\phi}_{13}^{-}=\psi_{13}^{-} \\
& \dot{\psi}_{13}^{-}=-\beta \psi_{13}^{-}-\cos \phi_{13}^{+} \sin \phi_{13}^{-}-\alpha_{s} \psi_{13}^{-}
\end{aligned}
$$

Linearizing eq. 4 we get the approximate dynamics transverse to the synchronization manifold. In terms of the Jacobian matrix we can rewrite the above equation as

$$
\left(\begin{array}{c}
\dot{\phi}_{1,3}^{-} \\
\dot{\psi}_{1,3}^{-}
\end{array}\right)=\left(\begin{array}{cc}
0 & 1 \\
\cos \phi_{1} & -\beta-\alpha_{s}
\end{array}\right)\left(\begin{array}{c}
\phi_{1,3}^{-} \\
\psi_{1,3}^{-}
\end{array}\right)
$$

where $\sin \phi_{1,3}^{-} \approx \phi_{1,3}^{-}$and $\cos \phi_{1,3}^{+} \approx \cos \phi_{1}$ as $\phi_{1} \approx \phi_{3}$ in the synchronization manifold. The eigen values of the matrix are

$$
m_{1,2}=-\frac{\left(\alpha_{s}+\beta\right)}{2}\left[1 \pm \sqrt{1+\frac{4 \cos \phi_{1}}{\left(\alpha_{s}+\beta\right)^{2}}}\right]
$$


The stability of the synchronous state is controlled by the eigen values $m_{1,2}$ [22]. If $m_{1,2}$ are complex conjugates with negative real part, the corresponding solution is stable. In the above case the average of the term in the radical is found and it is a complex number with real part greater than unity. The real part of the largest eigen value is thus found to be negative and hence satisfy the criterion for stability of synchronization.

As a second test, we follow the method given by Landsman et.al [23] where the conditional Lyapunov exponents are calculated with respect to the perturbation out of the synchronization manifold. Eq. 3 reduces to a set of four equations in the synchronized state as the outer junctions may be represented by a single set of equations. In terms of the synchronous solutions $\phi(t)$ and $\psi(t)$, we can define variables $\Delta \phi(t)=\phi_{1}(t)-\phi(t)$ and $\Delta \psi(t)=\psi_{1}(t)-\psi(t)$. Linearizing transverse to the synchronization manifold, we have

$$
\frac{d \Delta \phi_{i}}{d t}=J \Delta \phi_{i}
$$

and

$$
\frac{d \Delta \psi_{i}}{d t}=J \Delta \psi_{i}
$$

where $\mathrm{i}=1,3$ and $J$ is the Jacobian matrix evaluated at $\Delta \phi(t)$ and $\Delta \psi(t)$. Thus we have

$$
\left(\begin{array}{c}
\Delta \dot{\phi}_{1,3} \\
\Delta \dot{\psi}_{1,3}
\end{array}\right)=\left(\begin{array}{cc}
0 & 1 \\
1 & -\beta-\alpha_{s}
\end{array}\right)\left(\begin{array}{c}
\Delta \phi_{1,3} \\
\Delta \psi_{1,3}
\end{array}\right)
$$

$\Delta \phi_{1,3}$ and $\Delta \psi_{1,3}$ are the perturbations of the outer oscillators from the synchronous solution $\{\phi(t), \psi(t)\}$.

The Wronskian of the linearized system can be related to the trace of the matrix by the Abel's formula [23]

$$
W(t)=\left|\begin{array}{cc}
\Delta \phi & \Delta \psi \\
\Delta \dot{\phi} & \Delta \dot{\psi}
\end{array}\right|=\exp \left(\int_{0}^{t}\left(-\alpha_{s}-\beta\right) d t^{\prime}\right)
$$

where we have dropped the subscripts of the linearized variable. The Wronskian gives the phase space dynamics of the system. Taking the natural log of the Wronskian we get

$$
\ln [W(t)]=\ln |\Delta \phi \Delta \dot{\psi}-\Delta \psi \Delta \dot{\phi}|=-\int_{0}^{t}\left(\alpha_{s}+\beta\right) d t
$$

which is a monotonically decreasing function of time. The sum of the conditional Lyapunov exponents is given as,

$$
\sum_{j=1}^{M} \lambda_{j}=\lim _{t \rightarrow \infty} \frac{1}{t} \ln \mid \operatorname{det}\left(\Phi\left(\Delta \phi_{1,3}, \Delta \psi_{1,3}\right)(t) \mid\right.
$$


where $\Phi$ is the matrix solution of eqns. 6 and 7. The sum of the conditional Lyapunov exponents can be now approximated as

$$
\lambda_{1}+\lambda_{2} \approx-\left(\alpha_{s}+\beta\right)
$$

The sum of the conditional Lyapunov exponents is negative indicating that the phase space of the coupled system shrinks to a trajectory representing the synchronous solution. Thus the two methods lead to the same conclusion.
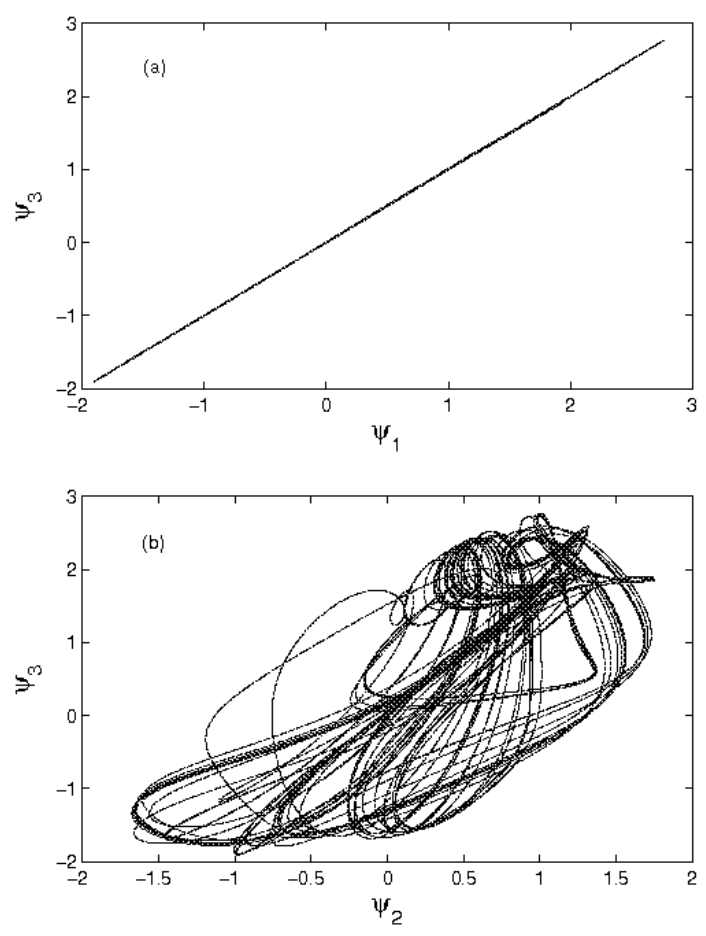

FIG. 2: (a) The outer juntions are synchronized (b) Outer junction and middle junction is uncorrelated. The parameter values are $\beta=0.3, i_{0}=1.2, \omega=0.6, i_{d c}=0.3, \alpha_{s}=0.37$.

Now we analyze the subsystem constituted by the outer and the middle junctions. We define new variables $\phi_{i 2}^{-}=\frac{\phi_{i}-\phi_{2}}{2}$ and $\psi_{i 3}^{-}=\frac{\psi_{i}-\psi_{2}}{2}$ where $i=1,3$. As the outer junctions are identical, it is enough to study any one subsystem. So considering the case with $i=1$, we write,

$$
\begin{aligned}
& \dot{\phi}_{12}^{-}=\psi_{12}^{-} \\
& \dot{\psi}_{12}^{-}=-\beta \psi_{12}^{-}-\cos \phi_{12}^{+} \sin \phi_{12}^{-}+\frac{1}{2}\left[i_{d c}+i_{0} \cos (\Omega t)\right]-\alpha_{s}\left(\frac{3}{2} \psi_{12}^{-}\right) .
\end{aligned}
$$

From Eq. 11 we conclude that in the presence of an external applied field it is not possible to synchronize all the three junctions due to the asymmetry induced by the applied fields. 
However in the absence of an external field, an identical solution can exist for all the three junctions. Extending the symmetry analysis to a system of N JJs coupled in parallell with
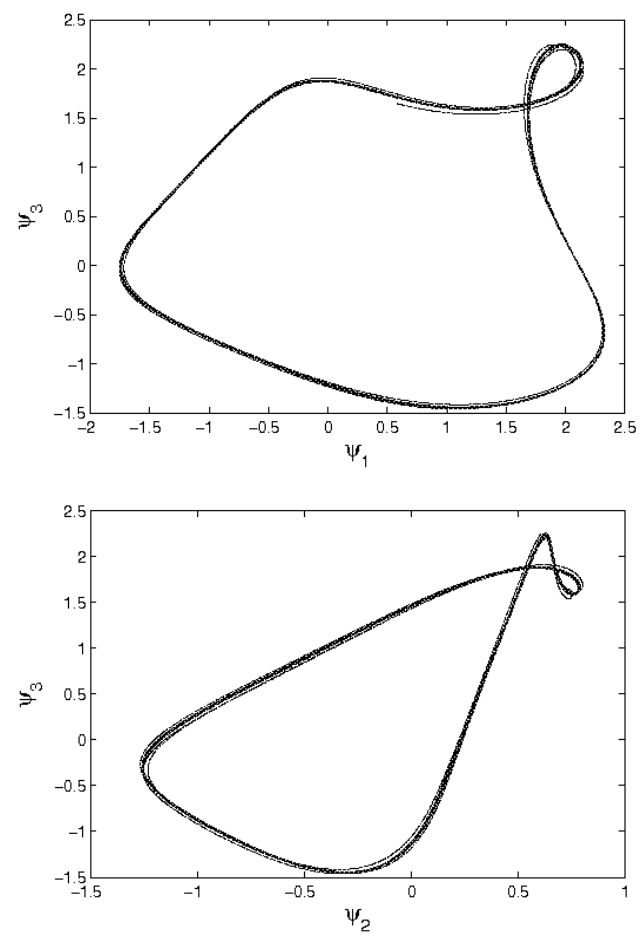

FIG. 3: (a) and (b) shows that the junctions are phase correlated. $\beta=0.3, i_{0}=1.2, \omega=0.6, i_{d c}=$ $0.3, \alpha_{s}=0.37$ and $\theta=0.5 \pi$.

nearest neighbour coupling, the second and the $(N-1)^{\text {th }}$ junction may have an identical solution for certain parameter values. Similarly, the third and the $(N-2)^{\text {nd }}$ junctions may have identical solutions and so on. Thus in the case of an array, from symmetry considerations we may deduce that $N / 2$ solutions may exist if there are even number of junctions in the array and $\frac{N+1}{2}$ solutions will be present for odd number of junctions. The time series plot for an array of 7 and 8 junction is plotted in Fig.4. It can be observed from Fig 4(a) that in an array of seven JJs the four solutions exists for the parameter range considered. The fourth junction has an independent solution. In Fig,4(b) we have plotted the time series for $8 \mathrm{JJs}$.

The presence of a phase difference between the applied fields changes the scenario completely. On the application of a small phase difference between the applied fields, the outer junctions desynchronize and all the three junctions are thus uncorrelated. But for sufficiently large values of phase differences, all the three junctions are found to be in phase synchro- 

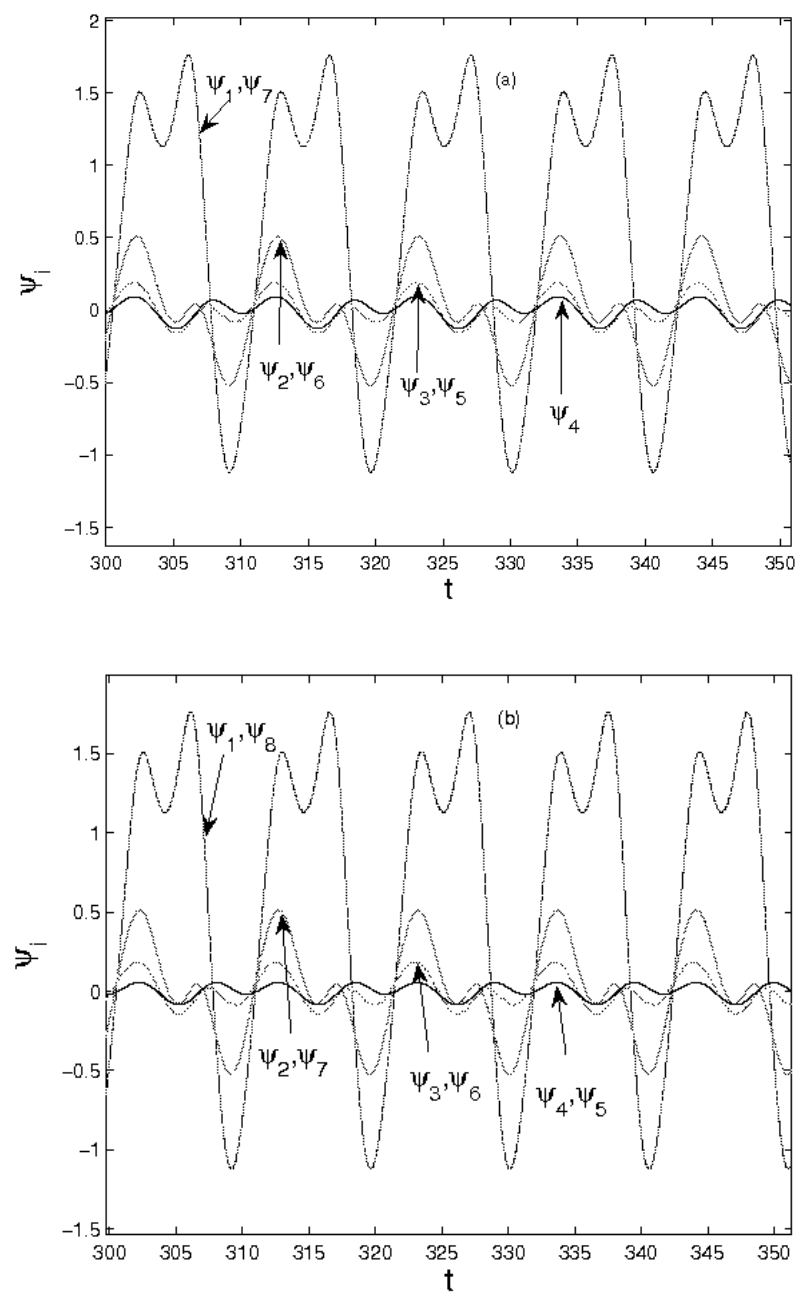

FIG. 4: (a)shows the time series plot for an array of 7 JJs and (b) for 8 junctions. $\beta=0.3, i_{0}=$ $1.2, \omega=0.6, i_{d c}=0.3, \alpha_{s}=0.37$ and $\theta=0.5 \pi$.

nization. Considering the difference variables $\psi_{1,2}, \psi_{1,3}, \psi_{3,2}$ as defined earlier, we explain the phenomena as follows. Due to the asymmetry that arises between the outer junctions in the presence of the phase difference we need the extra variable $\psi_{3,2}$ to analyze this situation. The equations for the three difference variables may be written by substituting eq as

$$
\begin{aligned}
& \dot{\psi}_{12}^{-}=-\beta \psi_{12}^{-}-\cos \phi_{12}^{+} \sin \phi_{12}^{-}+\frac{1}{2}\left[i_{d c}+i_{0} \cos (\Omega t)\right]-\alpha_{s}\left(\frac{\psi_{12}^{-}}{2}+\psi_{32}^{-}\right) \\
& \dot{\psi}_{13}^{-}=-\beta \psi_{13}^{-}-\cos \phi_{13}^{+} \sin \phi_{13}^{-}+i_{0}^{\prime} \sin \left(\Omega t+\frac{\theta}{2}\right)-\alpha_{s}\left(\psi_{13}^{-}-\psi_{32}^{-}\right) \\
& \dot{\psi}_{32}^{-}=-\beta \psi_{32}^{-}-\cos \phi_{32}^{+} \sin \phi_{32}^{-}+\frac{1}{2}\left[i_{d c}+i_{0} \cos (\Omega t+\theta)\right]-\alpha_{s}\left(\frac{\psi_{32}^{-}}{2}+\psi_{12}^{-}\right)
\end{aligned}
$$

where $i_{0}^{\prime}=i_{0} \sin \frac{\theta}{2}$. Thus each subsystems experiences a different driving field with the same 
frequency but different phases. Due to the phase relationship between the driving fields, a definite phase relationship is found to exist between all three junctions.

The level of mismatch of chaotic synchronization can be given quantitatively by taking the similarity function $S(\tau)$ as a time averaged difference between the variables $\psi_{i}$ taken with time shift $\tau[11]$

$$
S^{2}(\tau)=\frac{\left\langle\left[\psi_{1}(t+\tau)-\psi_{2}(t)\right]^{2}\right\rangle}{\left[\left\langle\psi_{1}^{2}(t)\right\rangle\right]\left[\left\langle\psi_{2}^{2}(t)\right\rangle\right]^{1 / 2}}
$$

and

$$
S^{2}(\tau)=\frac{\left\langle\left[\psi_{1}(t+\tau)-\psi_{3}(t)\right]^{2}\right\rangle}{\left[\left\langle\psi_{1}^{2}(t)\right\rangle\right]\left[\left\langle\psi_{3}^{2}(t)\right\rangle\right]^{1 / 2}} .
$$

and searching for its minimum $\sigma=\min _{\tau} S(\tau)$. If $\psi_{1}(t)=\psi_{3}(t)$, then $S(\tau)$ has a minimum value $\sigma=0$ for $\tau=0$. If both $\psi_{1}(t)$ and $\psi_{3}(t)$ are independent then $S(\tau) \approx 1$ for all the time. Line 1 in Fig. 5 shows complete synchronization between the end junctions and line 2 shows that the outer and middle junctions are desynchronized when no phase difference is present. A minimum of $S(\tau)$ indicates the the existence of a time shift between the two variables related to the phase shift. The amplitudes are uncorrelated in this regime, but phase correlation is present as indicated by lines 3 and 4 in the presence of a phase difference between the applied fields. On the application of a phase difference of $\pi / 2$ the dynamics
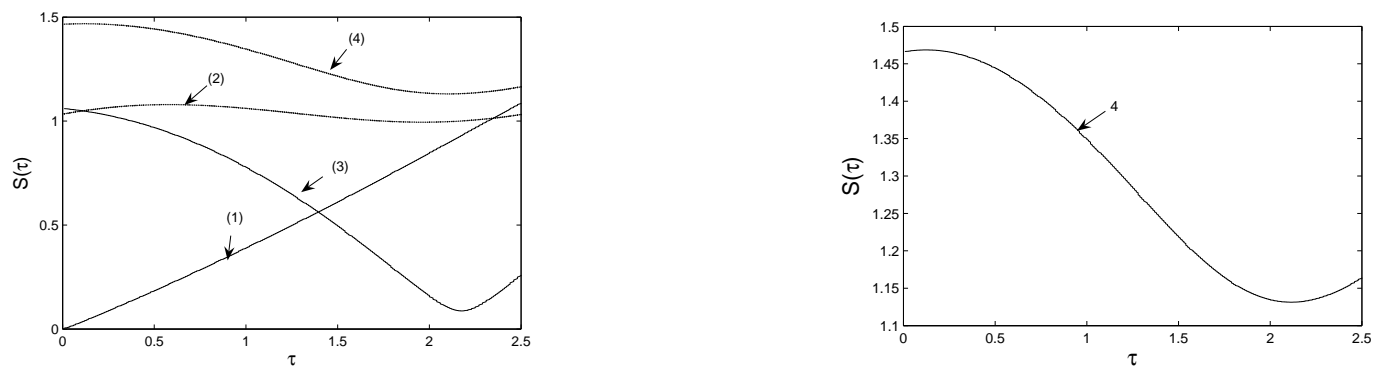

FIG. 5: (1) is $S(\tau)$ for variables $\psi_{1} \& \psi_{3},(2)$ for $\psi_{1} \& \psi_{2}$. Line (1) shows complete synchronization while the other is not synchronized. Both these lines are plotted with no phase difference applied (3) and (4) gives the similarity function for the variables $\psi_{1} \& \psi_{3}$ and $\psi_{1} \& \psi_{2}$ in the presence of phase difference $\theta=0.5 \pi$. The second figure shows line 4 where the dip can be observed clearly.

changes to periodic one as can be observed from Fig. 6 


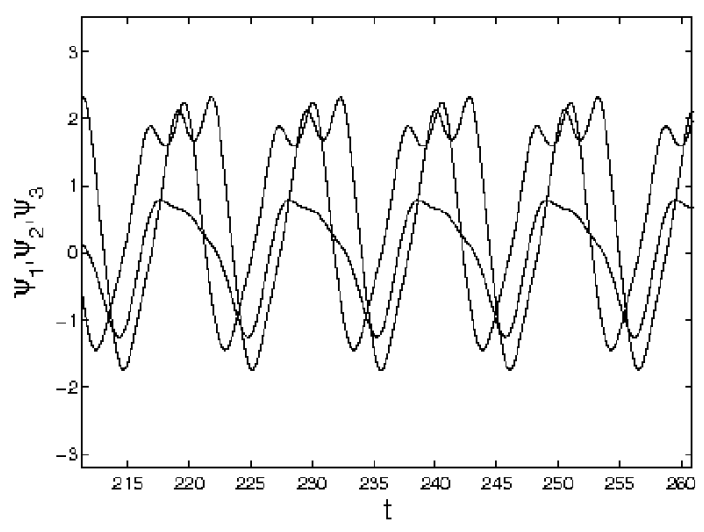

FIG. 6: The variables corresponding to the three JJs are ploted against time which indicates the periodic behavior.

\section{RESULT AND DISCUSSION}

We consider a parallel array of JJs with linking resistor $R_{s}$ and the conditions for synchronization is discussed. The outer junctions being symmetric, can possess identical solution and hence may synchronize depending on the parameter values. Linear stability analysis is done to find the stability of the synchronous solution of the outer junctions. The sum of conditional Lyapunov exponents calculated for the outer sub-system is found to be negative indicating stable synchronous state. From symmetry considerations we show that all three junctions could be synchronized only in the absence of an external field. Similarly in an array of $\mathrm{N}$ Josephson junctions, $N / 2$ identical solutions may exist if the number of junctions is even and $\frac{N+1}{2}$ solutions may exist if the number of junction is odd. In the presence of a small phase difference, the system desynchronizes due to the asymmetry induced by the phase difference. As the phase difference is increased, in the case of three junctions all the three junctions act as if they are driven by different driving fields having the same frequency, but different phases. A phase synchronization is observed between all the three junctions and the motion becomes periodic. Thus, suppression of chaos can be obtained in Josephson junction systems in the presence of a phase difference between the applied fields and this property may find applications in the working of devices constructed using JJs. 


\section{Acknowledgments}

The authors acknowledge DRDO, Government of India for financial assistance through a major research project.

[1] B. A. Huberman, J. P. Crutchfield and N. H. Packard, Appl. Phys. Lett.37, 750 (1980).

[2] D. D'Humieres, M. R. Beasley, B. A. Huberman, and A. Libchaber, Phys. Rev. A 26, 3483 (1982).

[3] Y. Braiman and I. Goldhirsch, Phys. Rev. Lett. 66, 2545 (1991).

[4] K. Fesser, A. R. Bishop and P. Kumar, Appl. Phys. Lett.43, 123 (1983).

[5] W. C. Schieve, A. R. Bulsara and E. W. Jacobs, Phys. Rev. A 37, 3541, (1988).

[6] Ezequiel N. Pozzo and Daniel Domnguez, Phys. Rev. Lett. 98, 057006, (2007).

[7] H. Schulze, R. Behr, F. Mller, and J. Niemeyer, Appl. Phys. Lett. 73, 996 (1998).

[8] P. Benz and C. A. Hamilton, Appl. Phys. Lett. 68, 3171 (1996).

[9] L. M. Pecora and T. L. Carroll, Phys. Rev. Lett. 64, 821 (1990).

[10] A. Pikovsky,M.Rosenblum and J.Kurths, Synchronization: A Universal concept in Nonlinear Science, Cambridge University press, Cambridge, 2001.

[11] M. G. Rosenblum, A. S. Pikovsky, and J. Kurths, Phys. Rev. Lett. 78, 4193(1997).

[12] J. R. Terry et.al, Phys. Rev. E 59, 4036 (1999).

[13] J. W. Shuai and K. W. Wong, Phys. Rev. E 57, 7002 (1998).

[14] M. S. Baptista, C. Zhou, and J. Kurths, Chinese Phys. Lett. 23, 560 (2006).

[15] J. Hale, Functional Differential Equations, Springer-Verlag, New York, 1971.

[16] L. M. Pecora and T. L. Carroll, Phys. Rev. Lett. 80, 2109 (1998).

[17] H.W.Yin, J.H.Dai and H.J. Zhang, Phys. Rev. E 58, 5683 (1998).

[18] K.-T. Kim, M.-S. Kim, Y. Chong and J. Niemeyer, Appl. Phys. Lett. 88, 062501 (2006).

[19] Chitra R Nayak and V.C. Kuriakose, Phys.Lett.A 365, 284 (2007).

[20] D. Chevriaux, R. Khomeriki, and J. Leon, Phys. Rev. B 73, 214516 (2006).

[21] J.A.Blackburn, G.L.Baker and H.J.T.Smith, Phys. Rev. B 62, 5931 (2000).

[22] U.E. Vincent et.al, Chaos 14, 1018 (2004).

S.L. Ross, Differential Equations, John Wiley \& Sons, Third Edition (1984). 
[23] A. S. Landsman and I. B. Schwartz, Phys. Rev. E 75, 026201 (2007). 